\title{
Fluctuaciones de presión en la base del pozo de oscilación del P.H. Zimapán Hgo., con diferentes arreglos estudiados en modelo hidráulico ante las condiciones mínimas de operación
}

\author{
Pressure Fluctuations on the Bed of Surge Tank at \\ the H.P. Zimapan, Hgo., with Different Arrangements Studied \\ on Hydraulic Model, with the Lowest Operation Conditions \\ H. Marengo-Mogollón \\ Facultad de Ingeniería, UNAM \\ E-mail:humberto.marengo@cfe.gob.mx \\ F.J. Ochoa-Álvarez \\ Comisión Federal de Electricidad. \\ E-mail:federico.ochoa@cfe.gob.mx \\ C. Cortés-Cortés \\ Comisión Federal de Electricidad. \\ E-mail:carlos.cortes01@cfe.gob.mx
}

(Recibido: agosto de 2007; aceptado: septiembre de 2008)

\section{Resumen}

En este artículo se comparan las fluctuaciones de presión en el pozo de oscilación del P.H. Zimapán Hgo., las cuales fueron estudiadas en modelo hidráulico al considerar dicho pozo ubicado en diferentes posiciones; lateralmente y sobre el eje de la conducción; pozo simple y con tubería de conexión; con y sin placa de orificio, para maniobras de rechazo y demanda de carga de las turbinas de generación con el nivel del agua correspondiente al NAMINO. Se determinó la eficiencia hidráulica comparándola con las otras opciones encontrándose que la mejor opción para el funcionamiento hidráulico es el pozo lateral, que además permite optimizar el procedimiento constructivo

Descriptores: Pozos de oscilación, modelo hidráulico, fluctuaciones de presión, eficiencia hidráulica.

\begin{abstract}
In this paper, the pressure fluctuations of the surge tank in the Zimapan Hydroelectric Project are compared in a hydraulic model. The shaft is located lateral, over the conduction tunnel and in the simple form (permitting the tunnel entering the shaft), with and without orifice plates taking into account the demand and supply condition of energy with the minimum level of water of the conduction. It was determined the hydraulic efficiency and it was found that it was the best constructive option.
\end{abstract}

Keywords: Pressure fluctuations, surge tank, orifice plates, the hydraulic efficiency.

\section{Introducción}

El P.H. Zimapán se construyó entre 1989 y 1994 sobre el río Moctezuma en el centro de México por parte de la Comisión Federal de Electricidad; durante el proceso de construcción se decidió colocar el pozo de oscilación de la conducción en forma lateral, con la premisa de que las presiones hidráulicas se transmiten de 
forma semejante a un pozo convencional durante las maniobras de rechazo y arranque de las unidades.

Desde el punto de vista constructivo, se logró independizar los frentes de trabajo del túnel y del pozo de oscilación sin que se tuviera mayor interferencia entre ellos, lo cual representó una gran ventaja en el procedimiento de construcción de este frente. Sin embargo, la inquietud en cuanto a su eficiencia hidráulica, quedó como una tarea pendiente a demostrarse.

Con base en lo anterior, se juzgó conveniente estudiar en un modelo físico las fluctuaciones de presión en la base del pozo de oscilación ubicado en diferentes posiciones; lateralmente-como se construyó-, sobre el eje de la conducción; con pozo simple y con tubería de conexión; con y sin placa orificio para maniobras de rechazo y toma de carga de las turbinas de generación, al considerar el nivel del agua del embalse al NAMINO, ya que representar el nivel del agua al NAMO escapa de los limites físicos de espacio en el laboratorio.

\section{Descripción del proyecto}

La obra de generación está integrada por una obra de toma tipo rampa con umbral de entrada a la elevación 1500 msnm, un túnel de conducción de sección tipo herradura de 4,70 m de diámetro y $20964 \mathrm{~m}$ de longitud. Al término del túnel de conducción se ha dispuesto un pozo de oscilación de $12 \mathrm{~m}$ de diámetro y $132 \mathrm{~m}$ de altura.

La conducción se complementa con una tubería de acero de 3,50 m de diámetro y $1080 \mathrm{~m}$ de longitud que trabaja a presión. En su parte final, antes de llegar a la casa de máquinas, se bifurca en 2 ramales de 2,10 m de diámetro cada uno, los cuales alimentan dos turbinas tipo Pelton de $146 \mathrm{MW}$ cada una.

El nivel mínimo de operación (NAMINO) corresponde a la elevación 1520 msnm en el embalse, se comunica con el pozo de oscilación a través del túnel de conducción, y el comportamiento conjunto para flujo permanente es como vasos comunicantes; ya que las pérdidas de carga en todo el túnel hasta el pozo son de 35,68 m que corresponden (figura 1) a un coeficiente de rugosidad de Manning en prototipo $n_{p}=0,0145$; además hay que sumar las pérdidas locales a las pérdidas por fricción.

Las pérdidas de carga calculadas en el proyecto se muestran en la tabla 1, que también señala el tramo de la conducción (hasta el cadenamiento $20+964,00$ es concreto) y el tramo de acero (hasta el cadenamiento $21+971,00)$.

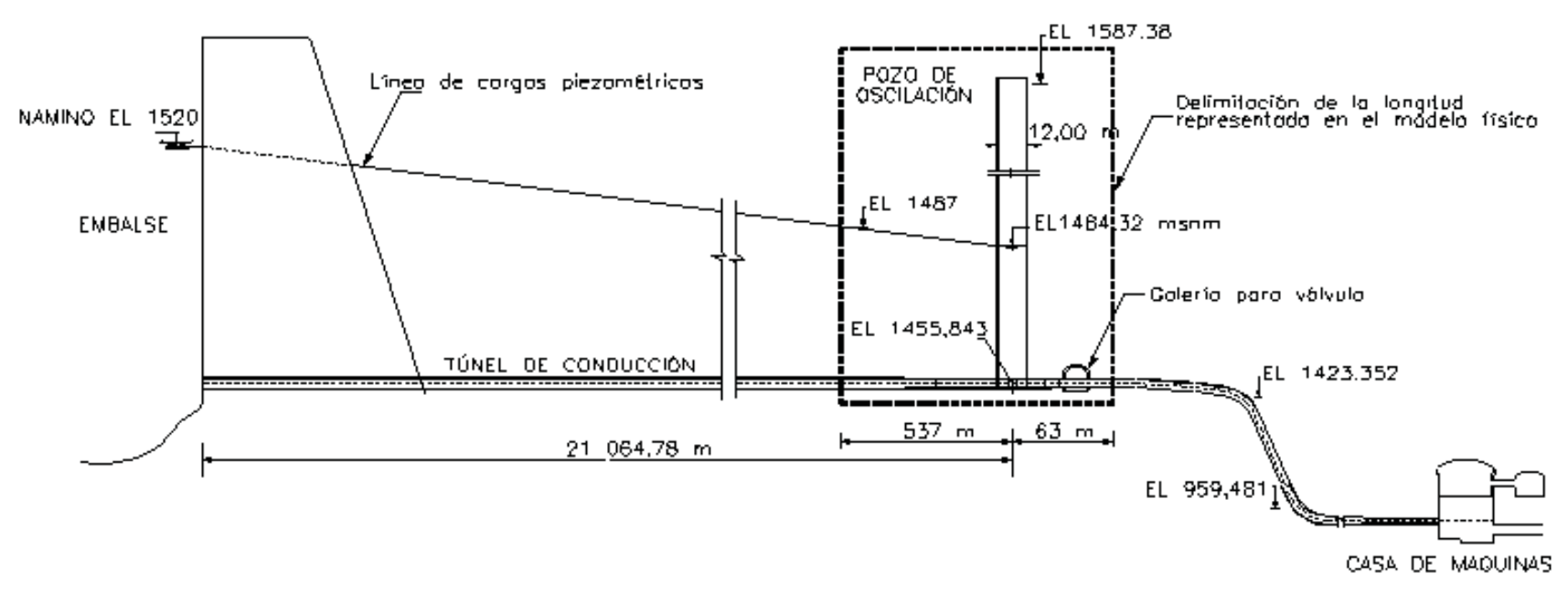

Figura 1. Esquema del túnel de conducción y longitud representada en el modelo físico 
DOI: http://dx.doi.org/10.22201/fi.25940732e.2009.10n4.029

H. Marengo-Mogollón, F.J. Ochoa-Álvarez y C. Cortés-Cortés

Tabla 1. Pérdidas de carga teóricas en el túnel de conducción

\begin{tabular}{ccccc}
\hline \multicolumn{5}{c}{ Conducción a presión } \\
\hline Tramo en $\mathrm{km}$ & Longitud en $\mathrm{m}$ & Espesor en pulg. & Diámetro en $\mathrm{m}$ & Pérdidas en $\mathrm{m}$ \\
\hline $0+000-20+964,00$ & 20964,00 & tunel - concreto & 4,70 & 34,168 \\
$20+964,00-21+064,00$ & 100,00 & $7 / 8$ " acero & 4,00 & 0,466 \\
$21+064,00-21+400,00$ & 336,00 & $3 / 4$ " acero & 3,50 & 2,250 \\
$21+400,00-21+447,00$ & 47,00 & $7 / 8 "$ " acero & 3,50 & 0,200 \\
$21+447,00-21+524,00$ & 77,00 & $1 "$ acero & 3,50 & 0,380 \\
$21+524,00-21+571,00$ & 47,00 & $11 / 8$ " acero & 3,50 & 0,200 \\
$21+571,00-21+618,00$ & 47,00 & $11 / 4 "$ acero & 3,50 & 0,200 \\
$21+618,00-21+665,00$ & 47,00 & $13 / 8 "$ acero & 3,50 & 0,340 \\
$21+665,00-21+870,00$ & 205,00 & $11 / 2$ " acero & 3,50 & 0,990 \\
$21+870,00-21+917,00$ & 47,00 & $15 / 8$ " acero & 3,50 & 0,550 \\
$21+917,00-21+971,00$ & 54,00 & $23 / 4 "$ acero & 3,50 & 0,270 \\
Tramo de ajuste & 43,40 & $13 / 4 "$ acero & 3,50 & 0,952 \\
\hline
\end{tabular}

\section{Descripción del modelo}

En la selección de la escala del modelo, $\mathrm{L}_{\mathrm{e}}=30$, se tomó en cuenta el espacio disponible en el Laboratorio de Hidráulica de la CFE, así como el gasto de alimentación para los ensayes, el costo y tiempo de su construcción. De acuerdo con la similitud de Froude se obtuvieron los valores de las escalas siguientes:

$\begin{array}{ll}\quad \begin{array}{l}\text { Escala } \\ \text { Líneas }\end{array} & L_{e}=30 \\ \text { Velocidades } & V_{e}=L_{e}^{0,5}=5,48 \\ \text { Gastos } & Q_{e}=L_{e}^{2,5}=4929,50 \\ \text { Rugosidades } & n_{e}=L_{e}^{0,16}=1,72 \\ \text { Tiempos } & T_{e}=L_{e}^{0,5}=5,48 \\ \text { Pesos } & W_{e}=L_{e}^{3}=27000\end{array}$

Es importante mencionar que la mayor dificultad para el diseño del modelo de este tipo de estructuras, surge cuando el túnel es muy largo, ya que se requiere de una tubería de gran longitud para representarlo. En forma similar, se presentan problemas con pozos demasiado altos. En este caso, conviene distorsionar la longitud del túnel respecto a la dimensión del pozo, resultando que el diámetro del túnel en el modelo puede ser semejante al diámetro del pozo.
En el caso de diseñar un modelo distorsionado, se debe cumplir satisfactoriamente la ley de semejanza en la cantidad de movimiento, así como las pérdidas entre modelo y prototipo. Sin embargo, por otro lado se recomienda (Berezowsky et al.,, 1988), que para simular con precisión las pérdidas locales y la unión del pozo con el túnel, el modelo debe construirse sin distorsión.

Considerando lo anterior y por limitaciones de espacio en el laboratorio, se decidió diseñar un modelo físico, construido a una escala $L_{e}=30$, con el criterio de similitud de Froude, sin distorsión y representar solo $537 \mathrm{~m}$ de longitud de túnel de conducción aguas arriba del pozo y $63 \mathrm{~m}$ aguas abajo del mismo; así como el aceptar que los ensayes se realizarían únicamente para el nivel del agua al (NAMINO) "Nivel de Aguas Mínimas de Operación".

La disposición geométrica del pozo de oscilación en sus diferentes localizaciones se muestran en la figura 2.

Debe señalarse que no hay similitud entre las pérdidas de energía de un túnel revestido de concreto en prototipo que tiene rugosidad granular y uno de rugosidad ondular como es la lucita, cuyo factor de fricción depende del número de Reynolds; razón por la cual en el modelo se simularon básicamente los fenómenos transitorios con sus pérdidas locales, tratando de no inferir los resultados a consideraciones de pérdidas por fricción. 


\section{Instrumentación, puesta a punto para su operación}

La instrumentación del modelo físico consistió en instalar:

1.Celdas de presión (figura 2), en diferentes secciones con el objeto de registrar las fluctuaciones de presión simultánea pozo-túnel de conducción.

2. Un generador de transitorios para simular la ley de rechazo y toma de carga de las turbinas, formado por:

- Servo-motor y PLC con pantalla para modificar parámetros (electrónica del sistema), (figura 3).

- Computadora personal para la captura y almacenamiento de registros.

3. Sistema de Adquisición de Datos (SAD).
4.Tubos piezométricos para registrar el perfil hidráulico en el túnel de conducción.

Para iniciar la operación del modelo se establece el flujo permanente en la conducción con el gasto de diseño de generación. Con los valores medidos con los tubos piezométricos del flujo permanente se ajustaron las celdas de presión y se iniciaron los ensayes.

\section{Registro de fluctuaciones de presión}

Se realizaron varios ensayes para los siguientes arreglos de pozo de oscilación:

- Considerando el pozo de oscilación lateral con y sin placa de orificio (figura 4).

- Pozo de oscilación alineado con el túnel de conducción y diferentes placas de orificio (figura $5), y$

- Con un pozo de oscilación simple (figura 6).

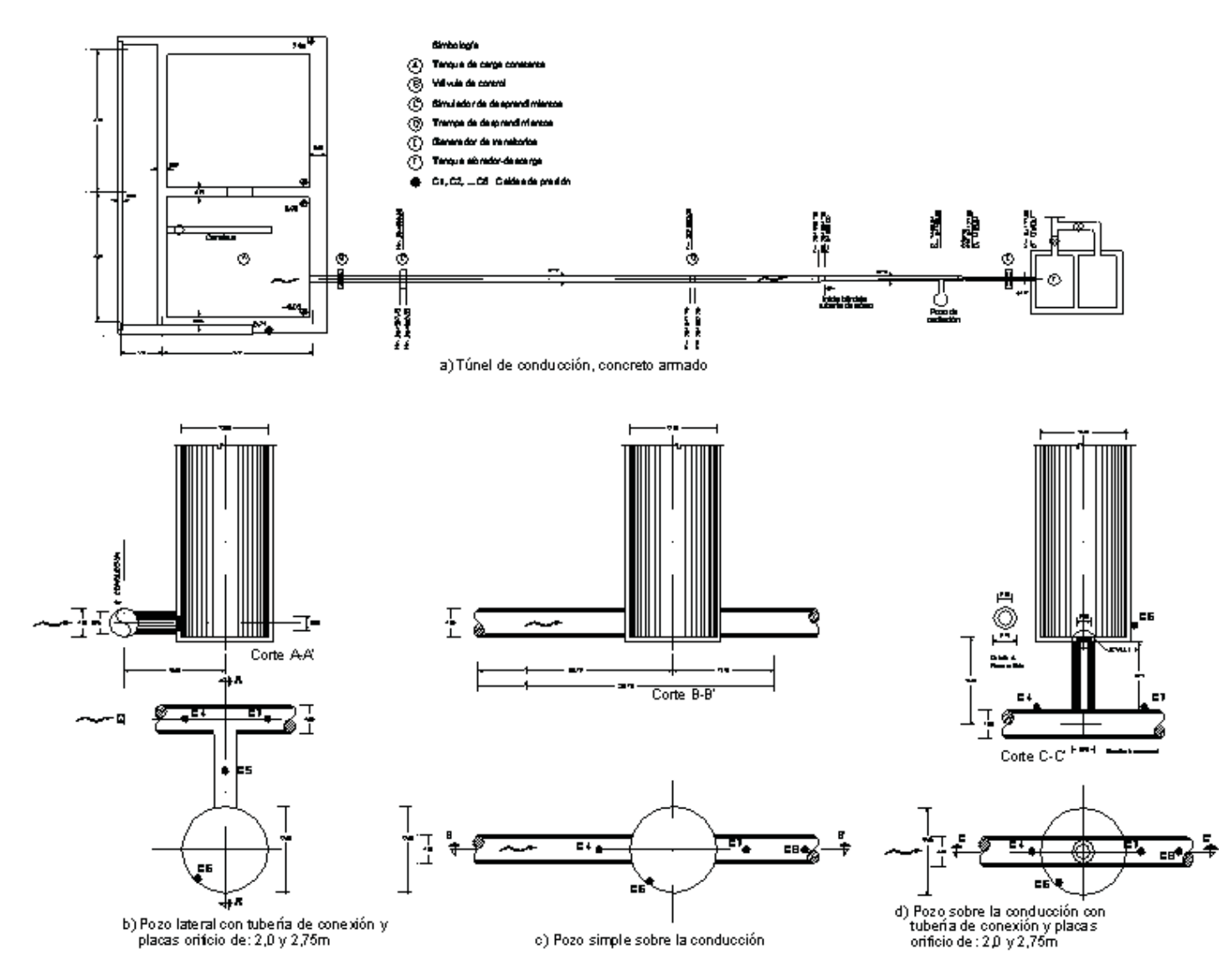

Figura 2. Esquema del modelo físico y ubicación de celdas de presión 


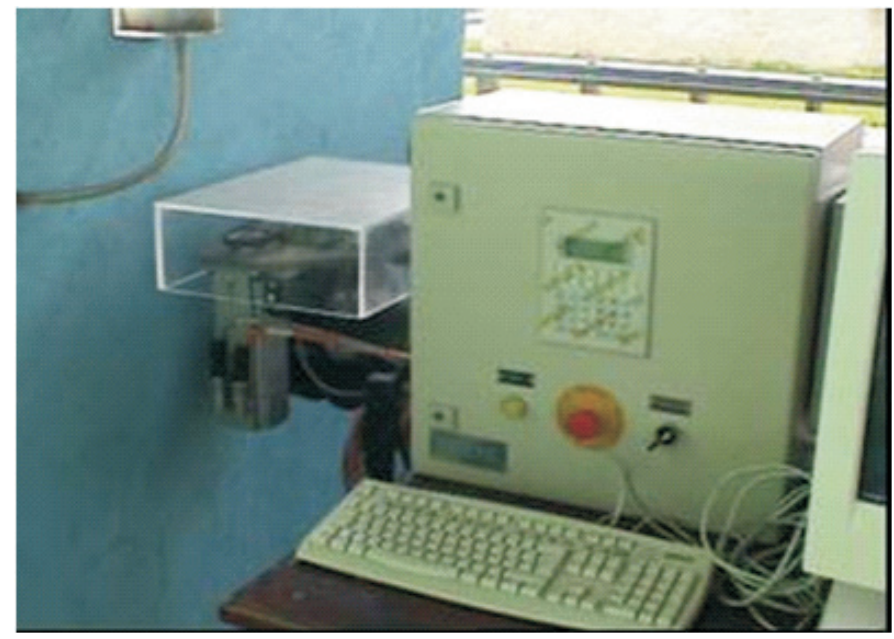

Figura 3. Servomotor y PLC de la instalación
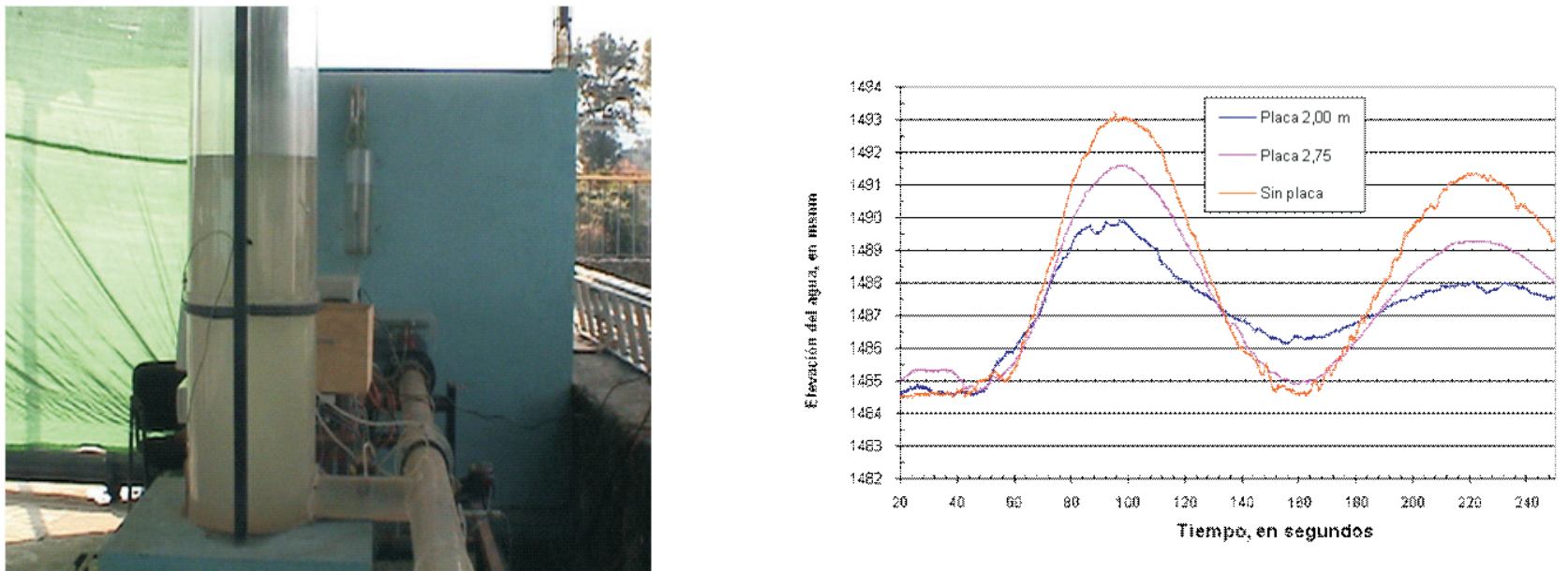

Figura 4. Fluctuaciones de presión con pozo de oscilación lateral y diferentes placas orificio
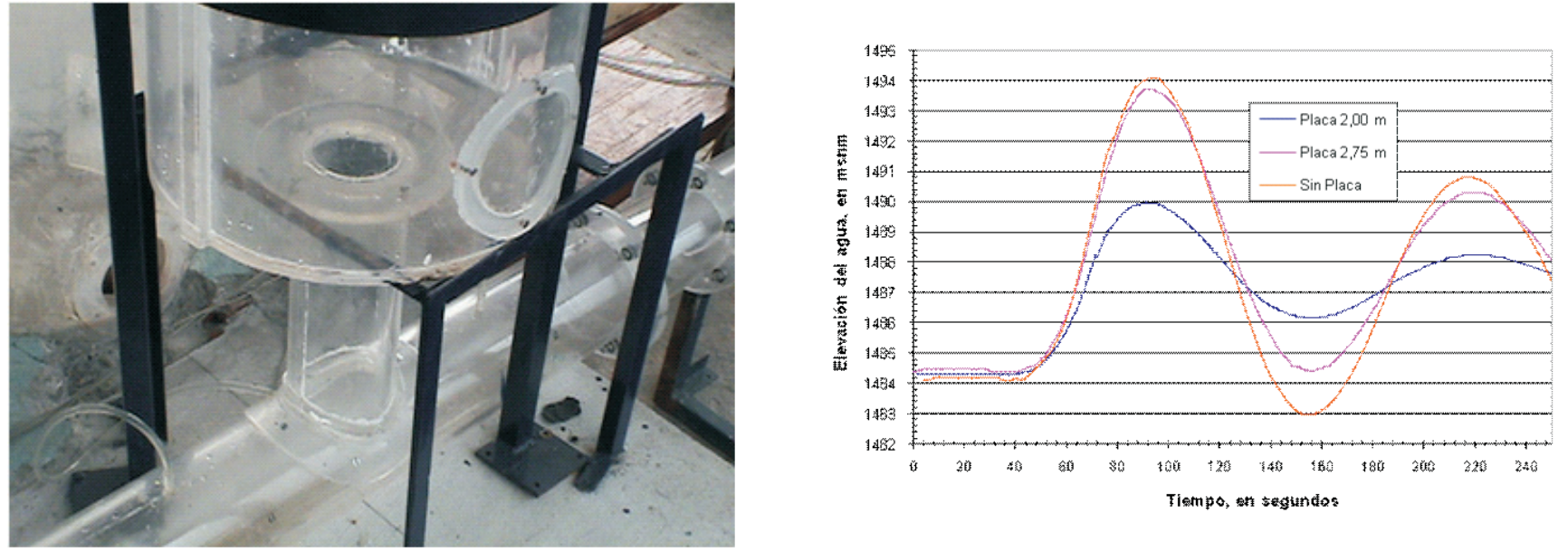

Figura 5. Fluctuaciones de presión con pozo de oscilación alineado con la conducción y diferentes placas de orificio 

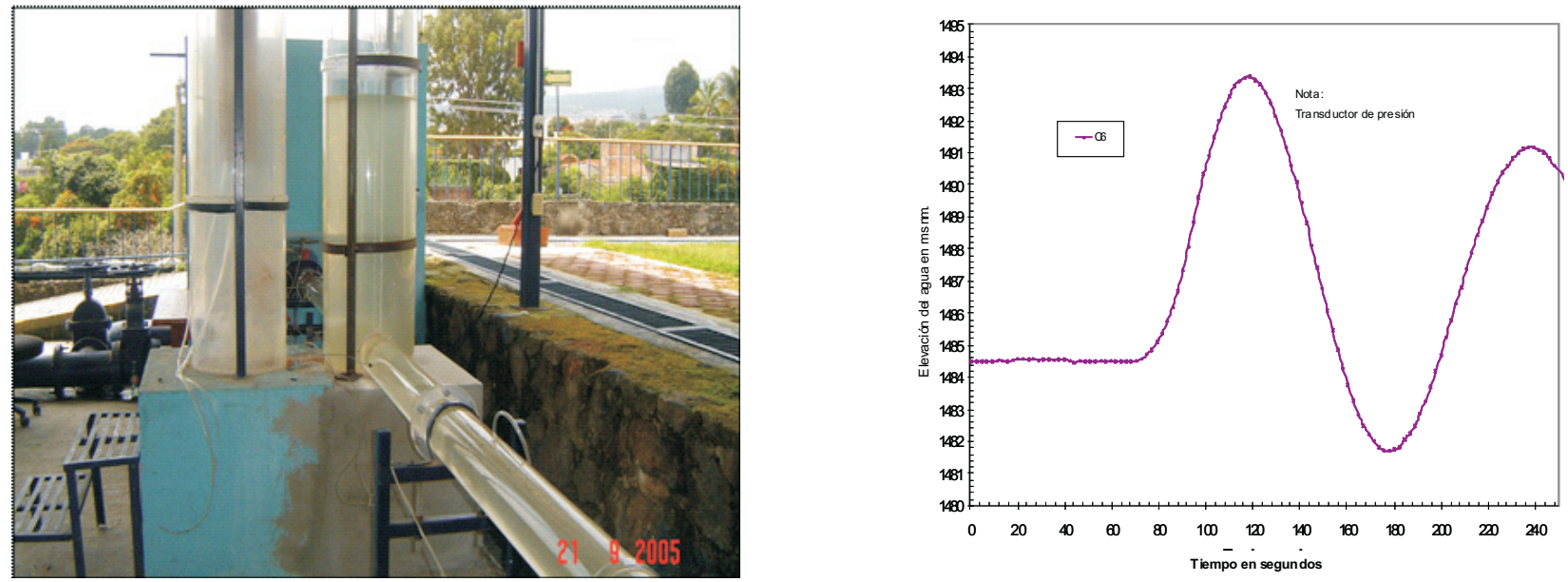

Figura 6. Fluctuaciones de presión con pozo de oscilación simple en la conducción

El nivel de agua en el embalse se reprodujo a la elevación 1520 msnm que corresponde al nivel de aguas mínimo ordinarias (NAMINO); al descontar las pérdidas de carga, se tiene al inicio del tramo modelado la elevación 1487 msnm y en el pozo de oscilación la elevación $1484,32 \mathrm{msnm}$.

\section{Análisis de resultados}

\section{Maniobra de rechazo de carga}

En cuanto a las maniobras de rechazo total de carga de las turbinas, al analizar los registros de fluctuaciones de presión para las diferentes ubicaciones del pozo de oscilación, se encontró que la disipación de la energía debida a la sobrepresión se traduce como una oscilación de masas en el pozo de oscilación y se transmite hacia el túnel de conducción en una cuantía que no pone en riesgo la estabilidad de la estructura.

Los niveles máximos y mínimos del agua registrados en el pozo de oscilación para el rechazo total de carga de las turbinas, ante condiciones extremas, fueron los señalados en la tabla 2.

Cabe aclarar que cuando se analizan los rechazos de carga, el diseño se hace considerando el embalse al NAMO (elevación 1560 msnm). Bajo esta condición, ante el accionamiento de los deflectores de las turbinas tipo pelton (y posteriormente el cierre de los inyectores de la rueda), el pozo no debe derramar y se buscó que la sobrepresión máxima no sobrepase un $15 \%$ de la carga

Tabla 2. Niveles máximos y mínimos del agua registrados en el pozo de oscilación para el rechazo total de carga de las turbinas

\begin{tabular}{ccccc}
\hline \multirow{2}{*}{ Localización del pozo } & Placa orificio en $\mathrm{m}$ & \multicolumn{3}{c}{ Niveles de agua en msnm } \\
\cline { 3 - 5 } & & Flujo permanente & Máximo & Mínimo \\
\hline \multirow{2}{*}{$\begin{array}{c}\text { Lateral con tubería } \\
\text { de conexión }\end{array}$} & 2,00 & 1484,57 & 1489,88 & 1484,57 \\
Simple en la línea de & 2,75 & 1485,05 & 1491,59 & 1484,66 \\
conducción & 3,20 (sin placa) & 1484,48 & 1493,04 & 1484,48 \\
Sin pozo & Sin placa & 1484,48 & 1493,36 & 1481,71 \\
$\begin{array}{c}\text { Sobre la conducción } \\
\text { con tubería } \\
\text { de conexión }\end{array}$ & 2,00 & 1484,64 & 1505,72 & 1483,59 \\
\hline
\end{tabular}


DOI: http://dx.doi.org/10.22201/fi.25940732e.2009.10n4.029

H. Marengo-Mogollón, F.J. Ochoa-Álvarez y C. Cortés-Cortés

estática máxima para no tener un espesor de placa en la tubería a presión muy grande.

En el caso del P.H. Zimapán, se cumplió plenamente con esta condición, ya que el nivel del agua máximo en el pozo, correspondió a la elevación 1582,38 msnm, que resulta en un $9,28 \%$ de sobrecarga, con respecto a la carga estática máxima que es de $603 \mathrm{~m}$. En la tabla 3 , se muestran los valores extremos registrados en cada celda de presión (figura 2) donde se aprecia la variación de la carga de presión que se traduce en una oscilación en masa para la conducción a presión. Las ondas de presión son reflejadas en el pozo hacia aguas abajo y una porción de estas ondas de presión se transmite hacia aguas arriba a lo largo del túnel de conducción.

La figura 7 muestra los valores máximo y mínimo de niveles de agua registrados en el pozo de oscilación considerando el rechazo de carga total de las turbinas.

\section{Maniobra de demanda de carga}

La condición analizada de máxima demanda de carga de las turbinas se realizó considerando el NAMINO, por lo que la oscilación mínima que se produce en el pozo-túnel de conducción ocurre en un tiempo muy corto, restableciéndose rápidamente el nivel de operación del sistema para la condición de flujo permanente. Los niveles del agua mínimos y máximos registrados en el pozo de oscilación para las diferentes condiciones geométricas del pozo, se indican en la tabla 4 , sin que se formen vórtices que produzcan inclusión de aire hacia la tubería a presión que modifiquen las condiciones normales de operación de las turbinas.

\section{Eficiencia del pozo para disipar energía}

Del análisis de la tabla 2 y la figura 7, la energía que se presenta en forma de carga de presión en el pozo de oscilación, es igual a la diferencia de las elevaciones $(1505,72-1484,64)=21,08 \mathrm{~m}$ columna de agua, es decir, es la diferencia de nivel del agua para flujo transitorio, menos el nivel del agua para flujo permanente. A partir de esta carga de presión sin pozo de oscilación, se tomó como base para determinar la eficiencia del pozo con sus diferentes placas orificio y sus localizaciones respecto al eje de la conducción con la siguiente expresión.

$$
\eta=\frac{E_{G}-E_{D}}{E_{G}} \times 100
$$

Donde:

$\mathrm{E}_{\mathrm{G}}$, oscilación máxima registrada sin pozo, en metros columna de agua; igual a $\left(\mathrm{E}_{\mathrm{ps}}-\mathrm{E}_{\mathrm{Ms}}\right)$,

$\mathrm{E}_{\mathrm{ps}}$, nivel máximo del agua registrado para flujo transitorio sin pozo;

$\mathrm{E}_{\mathrm{Ms}}$, nivel del agua registrado para flujo permanente sin pozo.

$\mathrm{E}_{\mathrm{D}}$, oscilación máxima registrada con pozo, en metros columna de agua; igual a $\left(\mathrm{E}_{\mathrm{p}}-\mathrm{E}_{\mathrm{M}}\right)$,

$\mathrm{E}_{\mathrm{p}}$, nivel máximo del agua registrado para flujo transitorio con pozo;

$\mathrm{E}_{\mathrm{M}}$, nivel del agua registrado para flujo permanente con pozo.

Tabla 3. Valores extremos de presión registrados en el pozo de oscilación ante rechazo de carga en las turbinas

\begin{tabular}{cccccccccc}
\hline Ensaye & \multicolumn{7}{c}{ Celda } \\
\hline & 1 & 2 & 3 & 4 & 5 & 6 & 7 & 8 & Localización \\
\hline 1 & 1488,282 & 1491,056 & 1491,758 & 1494,657 & 1494,294 & 1489,881 & 1494,952 & 1495,566 & Pozo \\
3 & 1487,529 & 1490,102 & 1490,032 & 1492,217 & 1492,244 & 1491,582 & 1492,500 & 1492,932 & lateral \\
5 & 1488,044 & 1490,399 & 1490,881 & 1493,152 & 1493,240 & 1493,043 & 1493,252 & 1493,120 & \\
7 & 1490,011 & 1496,551 & 1497,594 & 1503,983 & 1504,347 & & 1504,637 & 1506,515 & Sin pozo \\
9 & 1488,042 & 1488,857 & 1490,911 & 1493,256 & & 1493,359 & 1493,496 & 1493,512 & Pozo simple \\
11 & 1488,119 & 1492,015 & 1492,344 & 1495,979 & & 1489,9821 & 1496,602 & 1497,524 & Pozo sobre \\
13 & 1488,097 & 1489,583 & 1490,685 & 1492,496 & & 1493,706 & 1492,548 & 1493,094 & la \\
15 & 1487,897 & 1490,778 & 1491,132 & 1493,719 & & 1494,099 & 1493,431 & 1493,828 & conducción \\
Flujo & 1486,15 & 1485,74 & 1485,65 & 1484,47 & 1484,65 & 1484,57 & 1484,42 & 1483,39 & Pozo lateral \\
permanente & & & & & & & & &
\end{tabular}


DOI: http://dx.doi.org/10.22201/fi.25940732e.2009.10n4.029

Fluctuaciones de presión en la base del pozo de oscilación del P.H. Zimapán Hgo., con diferentes arreglos estudiados en modelo hidráulico ...

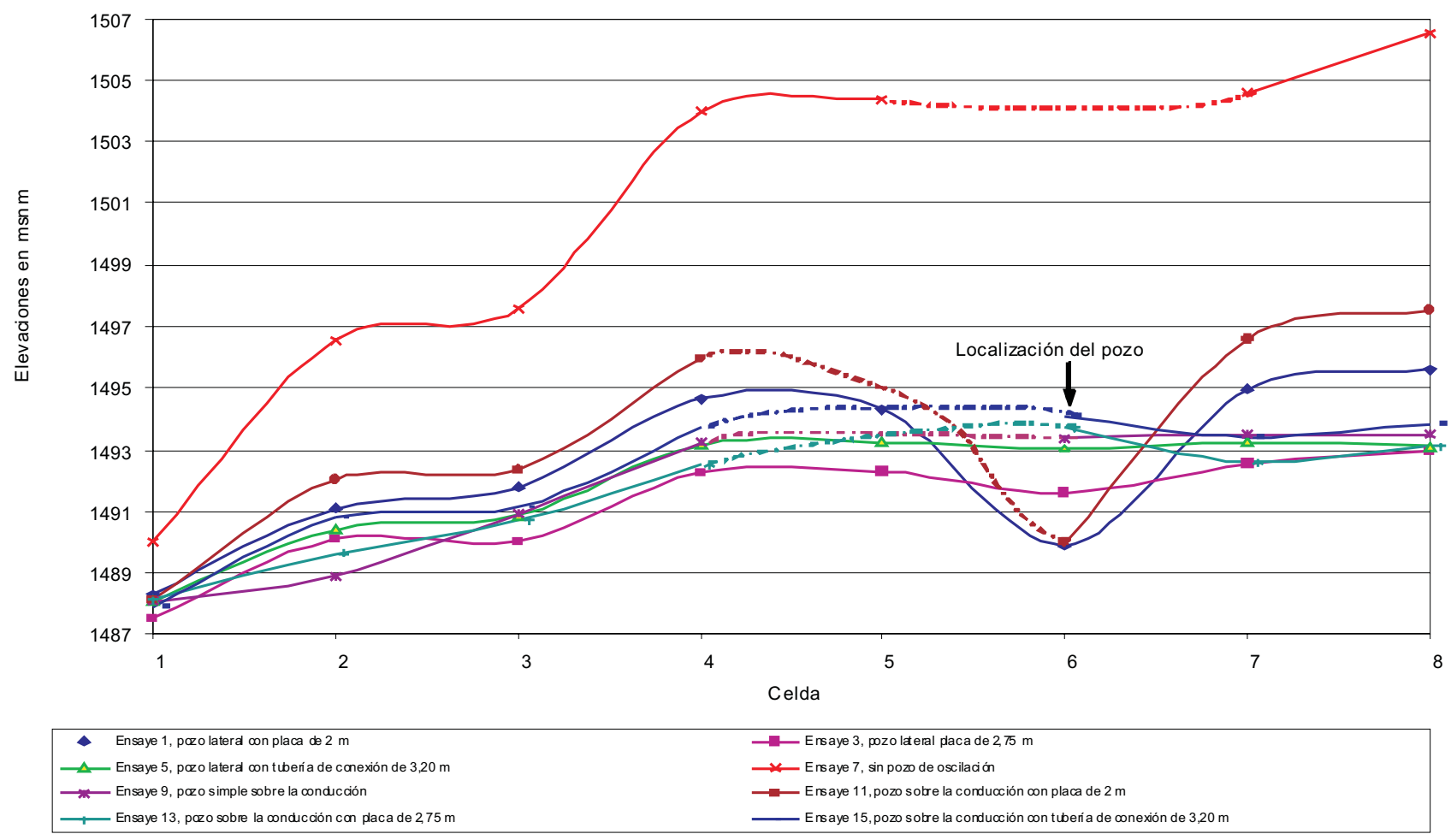

Figura 7. Valores máximo y minimo de niveles de agua registrados en el pozo de oscilación considerando el rechazo de carga total de las turbinas

Tabla 4. Niveles del agua mínimos y máximos registrados en el pozo de oscilación para las diferentes condiciones geométricas del pozo

\begin{tabular}{ccccc}
\hline $\begin{array}{c}\text { Localización } \\
\text { del pozo }\end{array}$ & $\begin{array}{c}\text { Placa orificio } \\
\text { en } \mathrm{m}\end{array}$ & Flujo permanente & \multicolumn{2}{c}{ Ninvel del agua en m } \\
\cline { 4 - 5 } & 2,00 & 1487,43 & Mínimo & Máximo \\
\hline $\begin{array}{c}\text { Lateral con } \\
\text { tubería } \\
\text { de conexión }\end{array}$ & 2,75 & 1487,42 & 1483,88 & 1488,10 \\
Simple en la & 3,20 (sin placa) & 1487,53 & 1482,62 & 1487,93 \\
conducción & Sin placa & 1487,14 & 1482,62 & 1487,19 \\
Sin pozo & Sin placa & 1487,64 & 1483,96 & 1487,82 \\
$\begin{array}{c}\text { Sobre la } \\
\text { conducción con } \\
\text { tubería de } \\
\text { conexión }\end{array}$ & 2,00 & 1487,40 & 1483,36 & 1487,57 \\
\hline
\end{tabular}


DOI: http://dx.doi.org/10.22201/fi.25940732e.2009.10n4.029

H. Marengo-Mogollón, F.J. Ochoa-Álvarez y C. Cortés-Cortés

Tabla 5. Eficiencia de los pozos de oscilación probados con distintas placas de orificio

\begin{tabular}{ccccc}
\hline \multirow{2}{*}{$\begin{array}{c}\text { Localización } \\
\text { del pozo }\end{array}$} & $\begin{array}{c}\text { Placa orificio } \\
\text { diámetro en } \mathrm{m}\end{array}$ & $\begin{array}{c}\text { Energía en } \mathrm{m} \\
\text { columna de agua }\end{array}$ & $\begin{array}{c}\text { \% disipado } \\
\text { en el pozo }\end{array}$ & $\begin{array}{c}\text { \% que pasa } \\
\text { hacia el túnel }\end{array}$ \\
\hline \multirow{2}{*}{ Lateral } & 2,00 & 5,31 & 74,81 & 25,19 \\
& 2,75 & 6,54 & 68,97 & 31,03 \\
Simple sobre & 3,20 & 8,56 & 59,39 & 40,61 \\
$\begin{array}{c}\text { 1 a conducción } \\
\begin{array}{c}\text { Sobre la } \\
\text { conducción con }\end{array}\end{array}$ & 12,00 & 8,88 & 57,87 & 42,13 \\
$\begin{array}{c}\text { batería de } \\
\text { conexión }\end{array}$ & 2,00 & 5,68 & 73,06 & 26,94 \\
Sin pozo & 3,75 & 9,32 & 55,79 & 44,21 \\
\hline
\end{tabular}

Por ejemplo, en el caso de pozo lateral con placa orificio de $2 \mathrm{~m}$ de diámetro, la eficiencia se estimó como:

$$
\begin{aligned}
\eta= & ((1505,72-1484,64)-(1489,88-1484,57) / \\
& (1505,72-1484,64)) * 100=74,81 \% ;
\end{aligned}
$$

de energía de presión que se libera y deja pasar el 25,19 $\%$. De manera análoga, se hizo para el resto de las placas orificio.

En la tabla 5 se presentan los valores comparativos.

De esta tabla 5 , se infiere que el pozo localizado lateralmente al eje del túnel de conducción con placa orificio de 2,00 m de diámetro es el más eficiente, convirtiendo en oscilación de masa la mayor parte de la energía, que deja pasar la menor cantidad de la onda de presión hacia el túnel de conducción.

La disposición geométrica anterior adoptada en el P.H. Zimapán, fue la mejor decisión, ya que hidráulicamente permite tener un funcionamiento hidráulico más eficiente y constructivamente permite tener frentes independientes del túnel de conducción. El haber elegido esta opción fue la mejor decisión, ya que desde el punto de vista hidráulico es la más eficiente y del constructivo, permite dos frentes de excavación independientes en el túnel de conducción.

\section{Conclusiones}

El pozo con arreglo lateral al eje del túnel de conducción con placa orificio de 2,00 m de diámetro es el más eficiente, haciendo oscilar la mayor parte de la columna de agua y dejar pasar la menor cantidad de carga de presión hacia el túnel de conducción.

La disposición geométrica adoptada en el P.H. Zimapán, fue la mejor decisión, ya que desde el punto de vista hidráulico es la mejor; y del constructivo, permite dos frentes de excavación independientes del túnel de conducción.

La magnitud de la energía que se transmite hacia el túnel de conducción en forma de onda de presión, genera un esfuerzo tangencial, que es inferior a la resistencia del concreto sometido a esfuerzos de tensión.

Para las condiciones de toma de carga de las turbinas, en condiciones de operación extrema, la oscilación máxima que se produce en el pozo-túnel de conducción se realiza en un tiempo muy corto, sin formación de vórtices que produzcan inclusión de aire hacia la tubería a presión, que modifiquen las condiciones normales de operación de las turbinas. Restableciéndose rápidamente el nivel de operación del sistema para la condición de flujo permanente.

\section{Referencia}

Berezowsky M. y Ramírez H. Cámaras de oscilación, análisis y diseño hidráulico. Series del Instituto de Ingeniería. Mayo, 1988.

\section{Bibliografía sugerida}

Camacho C.J.C. y Cortés C. Informe final del estudio del funcionamiento de la trampa de desprendimientos del túnel de 
DOI: http://dx.doi.org/10.22201/fi.25940732e.2009.10n4.029

Fluctuaciones de presión en la base del pozo de oscilación del P.H. Zimapán Hgo., con diferentes arreglos estudiados en modelo hidráulico ...

conducción de la C. H. Zimapán, en modelo físico. Laboratorio de Hidráulica. CFE. Diciembre, 2004.

CFE. Informe interno del recorrido de inspección en el túnel de conducción. CFE. Junio, 2002.

CFE. Manual de Diseño de Obras Civiles. Hidrotecnia. A 2.15. Técnicas Experimentales. CFE. 1981.

CFE. Manual de Diseño de Obras Civiles. Hidrotecnia. A 2.5. Cámaras de Oscilación. CFE. 1981.

CFE. Manual de Diseño de Obras Civiles. Hidrotecnia. A 2.6. Golpe de Ariete. CFE. 1981.

CFE. Manual de Diseño de Obras Civiles. Hidrotecnia. A.2.11. Hidráulica Fluvial. CFE. 1981.
Novak P. y Cabelka J. Models in Hydraulic Engineering, Physical Principles and Design Applications. Londres. Ed. Pitman. 1981.

Ochoa A.F.J. y Cortés C. Informe final del estudio de fluctuaciones de presión en el pozo de oscilación del túnel de conducción, en modelo hidráulico. Laboratorio de Hidráulica. CFE. Septiembre, 2006.

Nota: El Manual de Diseño de Obras Civiles de CFE, ya no se publica, aunque hay existencia en bibliotecas.

\section{Semblanza de los autores}

Humberto Marengo-Mogollon. Es ingeniero civil, maestro y doctor en ingeniería en hidráulica por la Facultad de Ingeniería de la UNAM. Desde 1980, ha participado en el diseño y construcción de proyectos hidroeléctricos. Actualmente funge como coordinador de proyectos hidroeléctricos. Ha recibido diversos reconocimientos como: la Medalla Gabino Barreda que otorga la UNAM a los mejores promedios en el doctorado, el Premio Nacional de Ingeniería Civil José A. Cuevas al mejor artículo técnico del periodo 1998-1999 y Miguel A. Urquijo al mejor artículo técnico del periodo 2000-2001 por parte del CICM, así como el Premio Nacional Ing. Francisco Torres H. a la práctica profesional de la hidráulica, en el año 2004, por la asociación mexicana de hidráulica y la Medalla Benito Juárez a la excelencia institucional en el año 2006 por la CFE. Asimismo, como académico titular en la academia de ingeniería, y como miembro regular de la academia mexicana de ciencias e investigador nacional (nivel I) del sistema nacional de investigadores. Desde 1985 es profesor de asignatura de la Facultad de Ingeniería de la UNAM.

Federico Joaquín Ochoa-Álvarez. Ingeniero civil por la Universidad Michoacana de San Nicolás de Hidalgo, cursó sus estudios de maestría en ingeniería hidráulica en la Facultad de Ingeniería de la Universidad Nacional Autónoma de México. Desempeñó funciones como responsable de agua en el Sistema de Administración Ambiental de la Secretaría del Medio Ambiente en el Gobierno del Distrito Federal. Ha sido responsable de estudio y supervisor técnico en algunos proyectos realizados en el laboratorio de hidráulica de la Coordinación de Proyectos Hidroeléctricos de la Comisión Federal de Electricidad. Actualmente se desempeña como encargado de área de modelos hidráulicos en el mismo laboratorio.

Carlos Cortés-Cortés. Egresado como ingeniero civil de la Escuela Superior de Ingeniería y Arquitectura del I.P.N. Obtuvo la maestría en ingeniería hidráulica en la División de Estudios de Posgrado de la Facultad de Ingeniería, UNAM. Tomó el diplomado en computación administrativa, así como cursos de informática y paquetería de PC's y electrónica, en el manejo de equipo de medición de alta precisión anemometría láser y cámara de alta velocidad. Inició sus actividades en el área de informática en la implantación del programa de información tecnológica de centrales generadoras en el Instituto de Investigaciones Eléctricas. A partir de 1989, en el Laboratorio de Hidráulica de la Comisión Federal de Electricidad ha participado en estudios experimentales de pozos de oscilación, vertedores escalonados, presas reguladoras, aireadores, desarrollo de proyectos de instrumentación de vertedores y obras de desvío en prototipo, cárcamos de bombeo, canales de llamada, desazolve de embalses y optimización de desarenadores, canales de descarga de plantas termoeléctricas y obras de toma de plantas hidroeléctricas. Asimismo, ha diseñado, construido y operado los modelos físicos de los proyectos antes mencionados. Actualmente es subjefe del laboratorio de hidráulica de la Comisión Federal de Electricidad, CFE. 